\title{
Hohe Herzfrequenz ist für jeden gefährlich
}

\author{
Auch für Gesunde gilt: Je höher die Herzfrequenz, umso schlechter die Prognose. Nicht nur \\ kardiovaskuläre, auch andere Krankheiten werden mit steigendem Ruhepuls häufiger.
}

Für eine Metaanalyse zur Bedeutung der Ruheherzfrequenz für fünf verschiedene Krankheitsgruppen wurden 45 Kohortenstudien mit repräsentativen Stichproben der Allgemeinbevölkerung berücksichtigt. Mit 18.364 Fällen bei 1,2 Millionen Probanden war die Datenlage für die koronare Herzkrankheit (KHK) am besten. Für nicht-kardiovaskuläre Erkrankungen ohne Karzinome gingen immerhin 2.174 Fälle bei 81.332 Patienten ein. Die Beobachtungsdauer lag zwischen 2 und 40 Jahren.

Erhöht sich der Ruhepuls um zehn Schläge pro Minute, steigt die Erkrankungshäufigkeit für KHK um 12\%, für Schlaganfall um 5\%, für plötzlichen Herztod um 12\% und für Malignome um 9\%. Nicht-kardiovaskuläre Erkrankungen kommen insgesamt um 16\% häufiger vor, solche ohne Malignome um 25\%. Alle Korrelationen sind signifikant. Beim Vergleich verschiedener Frequenzbereiche $(<60,60-70,70-80$, $>80 / \mathrm{min}$ ) ergibt sich ein linearer Zusammenhang mit der Morbidität.

- Zhang D, Wang W, Li F: Association between resting heart rate and coronary artery disease, stroke, sudden death and noncardiovascular diseases: a meta-analysis. CMAJ 2016, online 18. Oktober; doi:10.1503/cmaj.160050

\section{KOMMENTAR}

Es ist bekannt, dass Herz-Kreislauf-Erkrankungen und Gesamtmortalität mit dem Anstieg der Herzfrequenz zunehmen, auch bei Gesunden. Gängige Erklärungen sind hämodynamischer Stress sowie Erhöhung der kardialen Belastung und des Sauerstoffverbrauchs, die langfristig zu Myokardischämie und Arteriosklerose führen können.

Bislang berücksichtigen die Fachgesellschaften die Herzfrequenz nicht für die Berechnung des kardiovaskulären Risikos, da sie von einer Vielzahl von Ursachen wie Mentalität, Stress, Ängstlichkeit oder langfristiger körperlicher Aktivität beeinflusst wird. Außerdem korreliert sie mit diversen Erkrankungen, traditionellen Risikofaktoren und Entzündungsmarkern. Bisher konnte nicht gezeigt werden, dass eine Herzfrequenzsenkung bei kardial Gesunden z. B. mit Betablockern die Prognose verbessert.

Prof. Dr. med. H. Holzgreve

\section{Ein blinder Passagier verursacht üble Schmerzen}

Eine 36-jährige, bislang gesunde Frau litt seit zwei Tagen unter starken Schmerzen im Bereich von Thorax und Epigastrium, unter Übelkeit und Erbrechen. Das Abdomen war diffus druckschmerzhaft ohne Abwehrspannung oder Loslassschmerz. Sämtliche Laborbefunde inklusive der Herzmuskelenzyme waren normal. Auch

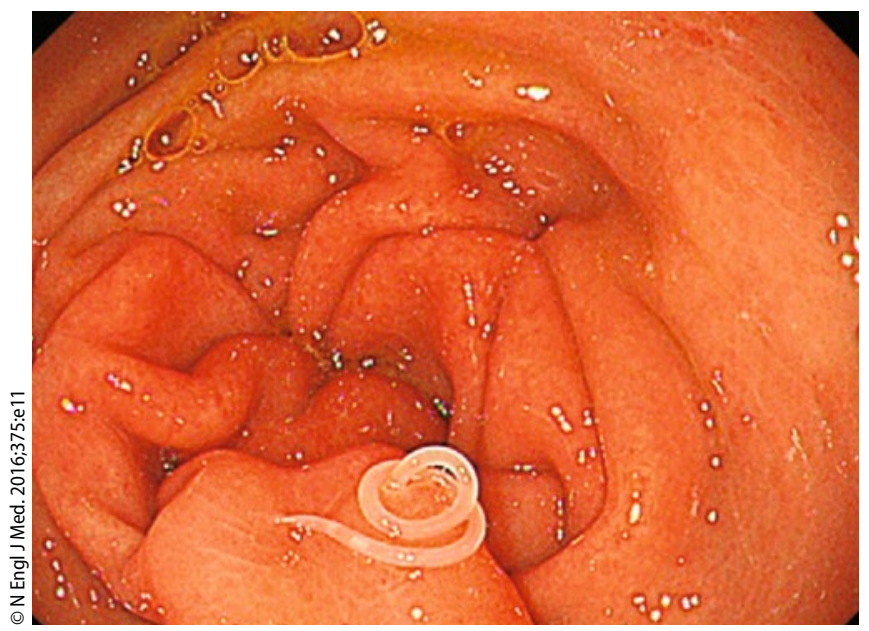

Fadenwurmlarve im Magen. in EKG und Röntgenthorax ergab sich kein pathologischer Befund. Die Patientin berichtete allerdings, zwei Stunden vor Einsetzen der Brustschmerzen rohen Lachs gegessen zu haben. Im Abdomen-CT zeigte sich eine diffuse Verdickung der Magenwand. Bei der anschließenden ÖGD erkannte man multiple Larven von Fadenwürmern, die sich in die Mukosa des gastroösophagealen Übergangs und praktisch aller Anteile des Magens gebohrt hatten (Abb.). Elf Larven wurden mit der Biopsiezange entfernt. Nach der ÖGD fühlte sich die Patientin bereits deutlich besser.

Die Anisakiasis wird von Fadenwürmern verursacht, die meist als Larven beim Genuss von rohem Fisch aufgenommen werden. Mit der Verbreitung von Sushi treten auch in Deutschland vermehrt Fälle auf. 12-24 Stunden nach der Infektion kommt es zu heftigen Bauchschmerzen, Schwindel, Übelkeit, Durchfall und Erbrechen. Im Extremfall können auch ein lleus oder eine Darmperforation auftreten. Die Erreger haben eine rund dreiwöchige Lebenszeit, sodass sich die Beschwerden in der Regel spontan zurückbilden. Der Erreger überlebt Temperaturen über $60^{\circ} \mathrm{C}$ oder unter $-20^{\circ} \mathrm{C}$ nicht. Die Infektion lässt sich vermeiden, wenn der rohe Fisch schockgefroren oder in Salzlake eingelegt wird.

Prof. Dr. med. H. S. FüeßI

- FuckizakiU, Nishikawa M (ufuchizaki@ybb.ne.jp). Gastric Anisakiasis. NEngl J Med. 2016;375:e11 